\title{
Estructura y función de los equipos de respuesta rápida para la atención de adultos en contextos hospitalarios de alta complejidad: Revisión sistemática de alcance
}

\section{Structure and roles of rapid response teams for adult care in high complexity hospitals: Scoping review

\begin{abstract}
Juliana Vanessa Rincón-López MD, MSc ${ }^{1,2}$; Diego Larrotta-Castillo MD ${ }^{1,2}$; Kelly Estrada-Orozco MD, MSc, PhD(c) ${ }^{1,2,3}$; Hernando Gaitán-Duarte, MD, MSc, ${ }^{1,2}$
\end{abstract}

Recibido: 16 de abril de 2021/Aceptado: 25 de junio de 2021

\section{RESUMEN}

Introducción y Objetivo: una importante proporción de pacientes hospitalizados presenta deterioro clínico severo que puede terminar en eventos adversos, paro cardíaco no esperado, o muerte; para reducir su frecuencia y prevenir sus consecuencias se han creado los equipos de respuesta rápida (ERR). El objetivo de esta revisión de alcance es describir la conformación, funcionamiento y resultados de la implementación de los ERR en el contexto hospitalario, con énfasis en los servicios de cirugía ginecológica y atención obstetricia.

Materiales y métodos: se llevó a cabo una búsqueda en las bases de datos de literatura médica Medline vía Pubmed, Embase vía OVID, LILACS, Cochrane Library y Open Gray. Se incluyeron estudios observacionales descriptivos y analíticos, estudios experimentales y estudios cualitativos que incluyeron ERR en instituciones de salud de alta complejidad u hospitales universitarios. Dos inves-

* Correspondecia: Grupo de evaluación de tecnologías y políticas en salud, Facultad de medicina, Universidad Nacional de Colombia, Carrera 30 No 45-03, edificio 471, oficina 231 Bogotá, Colombia. Tel. +57. Correo electrónico: julianav. rincon@gmail.com Grupo de Evaluación de Tecnologías y Políticas de Salud, Facultad de Medicina, Universidad Nacional de Colombia, Bogotá (Colombia).

1. Instituto de Investigaciones Clínicas, Facultad de Medicina, Universidad Nacional de Colombia, Bogotá (Colombia).

2. Grupo de Evaluación de Tecnologías y Políticas en Salud, Bogotá (Colombia) 3. Instituto de Evaluación Tecnológica en Salud, Bogotá (Colombia). tigadores seleccionaron los estudios y extrajeron los datos respecto a la conformación, funcionamiento, los criterios de activación del equipo, los tiempos de respuesta o las herramientas de evaluación de su desempeño. No se hicieron restricciones de fecha o estado de publicación. Se incluyeron estudios en inglés, español y portugués. Se hace síntesis narrativa de los hallazgos.

Resultados: la búsqueda arrojó 15,833 títulos, un total de 15 estudios cumplieron con los criterios de inclusión. Solo un estudio menciona el uso de los ERR en servicios de obstetricia. La conformación de los ERR es multidisciplinaria y están disponibles al menos 12 horas cada día. Sus funciones son la identificación temprana de pacientes con deterioro de la condición, especialmente en áreas por fuera de la unidad de cuidados intensivos y de pacientes con condiciones subyacentes o eventos desencadenantes que aumentan el riesgo de paro cardíaco. Además, implementan intervenciones rápidas multifacéticas que incluyen tratamientos farmacológicos, procedimientos cardiopulmonares, y desarrollan actividades de comunicación y formación. Se dispone de herramientas para la activación y evaluación de los procesos asistenciales. 
Conclusiones: la estructura y las funciones del ERR están claramente descritas, lo que permite que sean ensamblados en hospitales de alta complejidad. Se deben realizar más investigaciones sobre los beneficios y riesgos del uso de los ERR para mitigar los daños en pacientes con EREND y comparar la efectividad y seguridad entre la activación de códigos y las estrategias de ERR en los servicios de obstetricia.

Palabras clave: equipo hospitalario de respuesta rápida, gestión de riesgos, seguridad del paciente, puntuación de alerta temprana, deterioro clínico.

\section{ABSTRACT}

Introduction and Objective: A significant proportion of hospitalized patients experience severe clinical deterioration that may result in adverse events, unexpected cardiac arrest, or death. Rapid response teams (RRTs) have been created to reduce the frequency and prevent the consequences of these events. The objective of this scoping review is to describe the structure, role and results of the implementation of RRTs in the hospital context, with a focus on gynecological surgery and obstetric care.

Materials and methods: A search was conducted in the Medline via Pubmed, Embase via OVID, LILACS, Cochrane Library and Open Gray medical databases. The search included descriptive and analytical observational studies, experimental studies and qualitative studies that included RRTs in high complexity healthcare institutions or teaching hospitals. Two researchers selected the studies and extracted data pertaining to the structure, roles and team activation criteria, response times or tools to assess their performance. No date or publication status restrictions were applied. Studies in English, Spanish and Portuguese were included. A narrative synthesis of the findings is made.

Results: Overall, 15,833 titles were retrieved, of which 15 studies met the inclusion criteria. Only one study mentions the use of RRTs in obstetric services. RRTs have a multidisciplinary structure and they must be available at least 12 hours a day. The roles of RRTs include identification of patients who are deteriorating, especially outside the intensive care setting, and of patients with underlying conditions or triggering events that increase the risk of cardiac arrest. In addition, they implement rapid multifaceted interventions that include pharmacological treatments, cardiopulmonary procedures, and they develop communication and training activities. Tools for team activation and care process assessment are available.

Conclusions: The structure and roles of RRTs are clearly described, making it possible to assemble them in high complexity hospitals. Further research is required to explore risks and benefits of using RRTs to mitigate harm in patients with adverse events and to compare effectiveness and safety between code activation and RRT strategies in obstetrics services. Keywords: Hospital rapid response team, risk management, patient safety, early warning score, clinical deterioration.

\section{INTRODUCCIÓN}

El paro cardíaco no esperado y el deterioro del estado clínico son las principales causas de muerte en los pacientes hospitalizados. En Estados Unidos se ha descrito que cerca de 290,000 pacientes hospitalizados sufren paro cardíaco al año (1). Las principales causas de paro cardíaco son el infarto de miocardio, la arritmia o insuficiencia cardíaca (50\% - 60\%), seguidas de insuficiencia respiratoria causada por embolia pulmonar e hipoxemia generalizada (15\% 40\%) y las complicaciones vasculares neurológicas, la hemorragia y la sepsis (2). El paro cardíaco afecta a cerca del 4\% de los pacientes hospitalizados (3). Otros autores informan una frecuencia de 10 paros cardiacos intrahospitalarios por 1,000 admisiones (4).

Otras causas relevantes de muerte o paro cardíaco en pacientes hospitalizados son los eventos adversos, también conocidos como eventos reportables con efectos no deseados (EREND), definidos como el daño físico o psicológico no intencional que tiene un paciente como resultado de la prestación de atención 
médica o con motivo de ella (5). Se ha descrito que los EREND están relacionados con al menos 210,000 muertes por año en los Estados Unidos (6). En cuanto a la frecuencia, se ha descrito una incidencia acumulada que varía del 4\% en Estados Unidos (7) al 16\% en Australia (8), de los cuales hasta en un 65\% se consideraron evitables (9).

El deterioro del paciente también afecta a las pacientes sometidas a cirugía ginecológica y las mujeres gestantes (10). La enfermedad tromboembólica posterior a la cirugía pélvica mayor, el evento cerebrovascular asociado a la preeclampsia, la hemorragia y la sepsis, son las causas más comunes de paro cardíaco en la práctica obstétrica tanto en Canadá (11) como en Colombia $(12,13,14)$. Por otra parte, se ha informado que el 0,4\% de las gestantes sin morbilidad materna severa presentan muerte inesperada (11) por eventos no predecibles ni prevenibles como el embolismo amniótico (15). En Canadá, el paro cardíaco afecta a 1 de cada 12,500 partos (16). Respecto a los EREND en Colombia se ha descrito que estos afectan aproximadamente el 3,4\% de las gestantes (14), en Australia el $6 \%$, siendo prevenibles del 54 al 75\% de ellos $(8,17)$. De esta manera tanto los EREND como las complicaciones, pueden llevar a emergencias médicas. Estas se definen como ocasiones en las que un paciente se deteriora en su condición física o psicológica a tal punto que hay un riesgo inminente de daño serio (18, 19). Los profesionales de la salud han intentado reducir el impacto de las complicaciones por condiciones subyacentes o de los EREND mediante la identificación temprana de los pacientes que se encuentran en deterioro, basados en respuestas institucionales apropiadas como ocurre con los Equipos de Respuesta Rápida ERR (20).

En el año 2004, el Institute for Healthcare Improvement (IHI) propuso los ERR (Rapid Response Teams - RRT en inglés) o Equipos de Emergencia Médica - EEM (Medical Emergency Teams - MET) como una de las 6 intervenciones de la Campaña por 100,000 Vidas (100,000 Lives Campaign) (21). Sin embargo, los ERR fueron la única intervención que generó dudas sobre su efectividad después de la implementación (22).

\section{CONCEPTO}

Los ERR se definen como equipos multidisciplinarios que ofrecen apoyo oportuno en la valoración de pacientes con signos o síntomas de deterioro clínico, para evitar que ocurran lesiones, paros cardíacos, o fallecimientos (21). La evidencia sobre los beneficios de los ERR es controversial. Algunas revisiones sistemáticas muestran que los ERR no son efectivos para reducir las tasas de mortalidad en pacientes hospitalizados (23), y otras muestran que sus efectos no son consistentes a través de los desenlaces (24). Por otra parte, dos revisiones publicadas recientemente reportan beneficios en términos de mortalidad intrahospitalaria — RR 0.87, 95\% CI 0.81-0.95 (25); RR 0.88, 95\% CI: 0.83-0.93 (26) — y reducción de paros cardíacos en pacientes hospitalizados — RR 0.65, 95\% CI 0.61-0.70 (25); y RR 0.62, 95\% CI: 0.55-0.69 (26)—.

Con el fin de implementar ERR en una institución hospitalaria es necesario entender cómo se encuentran conformados y de qué forma interactúan sus equipos, las funciones asignadas y tiempos de respuesta que se requiere para atender a un llamado. Esta revisión sistémica de alcance tiene como objetivo identificar y sintetizar la evidencia disponible sobre la conformación y funcionamiento de los ERR en los hospitales de enseñanza o de alta complejidad que brindan atención a adultos. Al generar un resumen conceptual que examine las características de la evidencia disponible sobre los ERR, se podría informar y optimizar la implementación de los ERR en nuevos contextos.

\section{MATERIALES Y MÉTODOS}

Preguntas guía: ¿Cómo se conforman y funcionan los Equipos de Respuesta Rápida en hospitales de alta complejidad que atienden adultos?

Diseño: se realizó una revisión sistémica de alcance basada en la guía del Joanna Briggs Institute (27).

\section{Criterios para la selección de estudios}

Tipos de estudios. Ensayos clínicos aleatorizados en grupos, estudios cuasiexperimentales, estudios 
observacionales (cohortes, de corte transversal, casos y controles, reportes de casos) y estudios cualitativos. Tipo de participantes. Equipos de respuesta rápida ERR.

Contexto. Instituciones de atención en salud de alta complejidad u hospitales de enseñanza.

Concepto. Equipos de respuesta rápida que disminuyan los eventos no deseados en pacientes hospitalizados.

Desenlaces. Conformación: número de personas, profesión, funciones de miembros del equipo, experiencia. Funcionamiento: criterios de activación de los equipos, intervenciones, tiempos de respuesta y herramientas para el trabajo de los equipos.

\section{Métodos de búsqueda para la identificación de estudios}

La búsqueda de la literatura fue realizada en agosto de 2019 y se actualizó en mayo de 2021 en las siguientes bases de datos: Medline (vía Pubmed), Embase (vía OVID), LILACS y la Cochrane Library. Se consultó literatura gris mediante Open Grey. El algoritmo de búsqueda usado estuvo compuesto por términos libres e indexados de acuerdo con cada base de datos. No hubo restricciones de fecha o de estado de publicación. Se incluyeron estudios en inglés, español y portugués. La estrategia de búsqueda se muestra en materiales suplementarios (Apéndice 1).

\section{Selección de estudios y extracción de datos}

Un investigador (JRL) tamizó estudios por título y resumen buscando estudios potenciales. Los estudios que fueron seleccionados fueron tamizados en texto completo junto con otro investigador (DLC). Las dudas sobre la inclusión de algún estudio en las dos etapas descritas se resolvieron consultando un tercer evaluador (KEO) para llegar a un consenso. Dos autores extrajeron datos de manera independiente. Los datos extraídos relacionados con características de los estudios fueron: año de publicación, autores, diseño y país. Los relacionados con características de la institución donde se desarrolló el estudio fueron: nivel de complejidad, si se trataba o no de un hospital de enseñanza. Los relacionados con estructura del ERR fueron: número de miembros, perfil profesional, experiencia, funciones de cada miembro del equipo. Los relacionados con el funcionamiento fueron: disponibilidad del equipo en la institución, funciones del ERR, criterios de activación, personal responsable de la activación, intervenciones de los ERR, los tiempos de respuesta, tiempo de acción, presencia de líderes dentro del grupo, uso de herramientas especializadas para la valoración del paciente. El tiempo de respuesta se definió como el tiempo transcurrido desde la activación del ERR hasta la llegada a la cabecera del paciente. El tiempo de acción se definió como el tiempo que tomó el equipo para valorar al paciente y definir un tratamiento. Las herramientas de la implementación de los ERR se definieron como los algoritmos, protocolos, modelos y guías usadas en la atención del paciente, valoración clínica o procesos de activación dentro del ERR.

\section{Valoración de la calidad de los estudios incluidos}

La calidad metodológica de los estudios de cohorte se valoró usando la escala Newcastle Ottawa (NOS) (28). Para los estudios cualitativos y reportes de caso se utilizaron las herramientas de valoración crítica del Instituto Joanna Briggs (JBI) (29, 30). Los estudios transversales fueron evaluados utilizando la herramienta de Berra S. et al. (31). Para los estudios cuasiexperimentales se utilizó el instrumento de verificación de evaluación crítica del JBI (32), y para los estudios experimentales se utilizó la herramienta ROB (33).

\section{Análisis y síntesis de resultados}

Para la síntesis de la información se describen los valores relativos y absolutos de los estudios que responden a cómo están conformados los ERR. El análisis cualitativo se realizó mediante un enfoque descriptivo para obtener información detallada sobre la práctica en ERR y el contexto de aplicación. 
Los datos recolectados se sintetizaron en una tabla en la que se incluye la información encontrada en los estudios para cada categoría (características del estudio, estructurales del equipo de respuesta rápida, de la actuación y de la institución donde funciona el equipo).También se presenta la información en la modalidad de meta-resumen (34).

Para el reporte de los resultados y la elaboración del manuscrito se siguió la lista de chequeo de la extensión para revisiones de alcance PRISMA-ScR (35).

\section{RESULTADOS}

La búsqueda en las bases de datos arrojó 18,301 títulos, de los cuales 970 eran duplicados y se descartaron. Luego del tamizaje por título y abstract, 17284 títulos se eliminaron por no ajustarse a los criterios de inclusión. En total 47 estudios fueron tamizados en texto completo, de los cuales 15 cumplieron con los criterios de inclusión.

El proceso de selección de los estudios se muestra en un diagrama PRISMA (35) — Figura 1-.

\section{Figura 1.}

Diagrama PRISMA que resume el proceso de selección de estudios

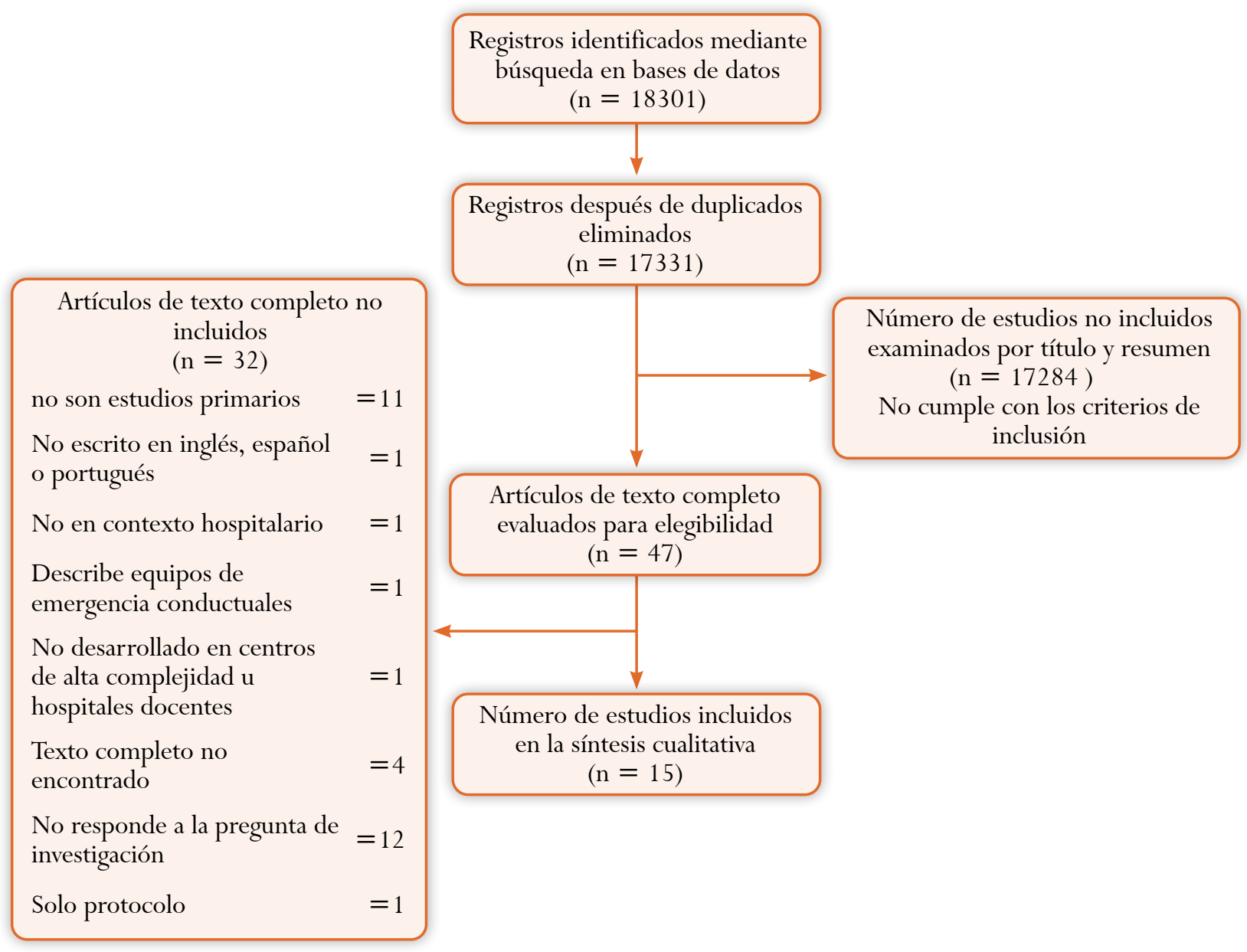

\section{Características de los estudios}

Respecto a los diseños de los estudios, tres tuvieron un diseño cualitativo $(36,37,38)$, uno tuvo un diseño cuasiexperimental (39), cuatro fueron re- porte de caso $(40,41,42,43)$, dos fueron estudios de corte transversal $(44,45)$, tres fueron cohortes retrospectivas $(46,47,48)$ y dos cohortes prospectivas $(49,50)$. Las características de los estudios se presentan en la Tabla 1. 


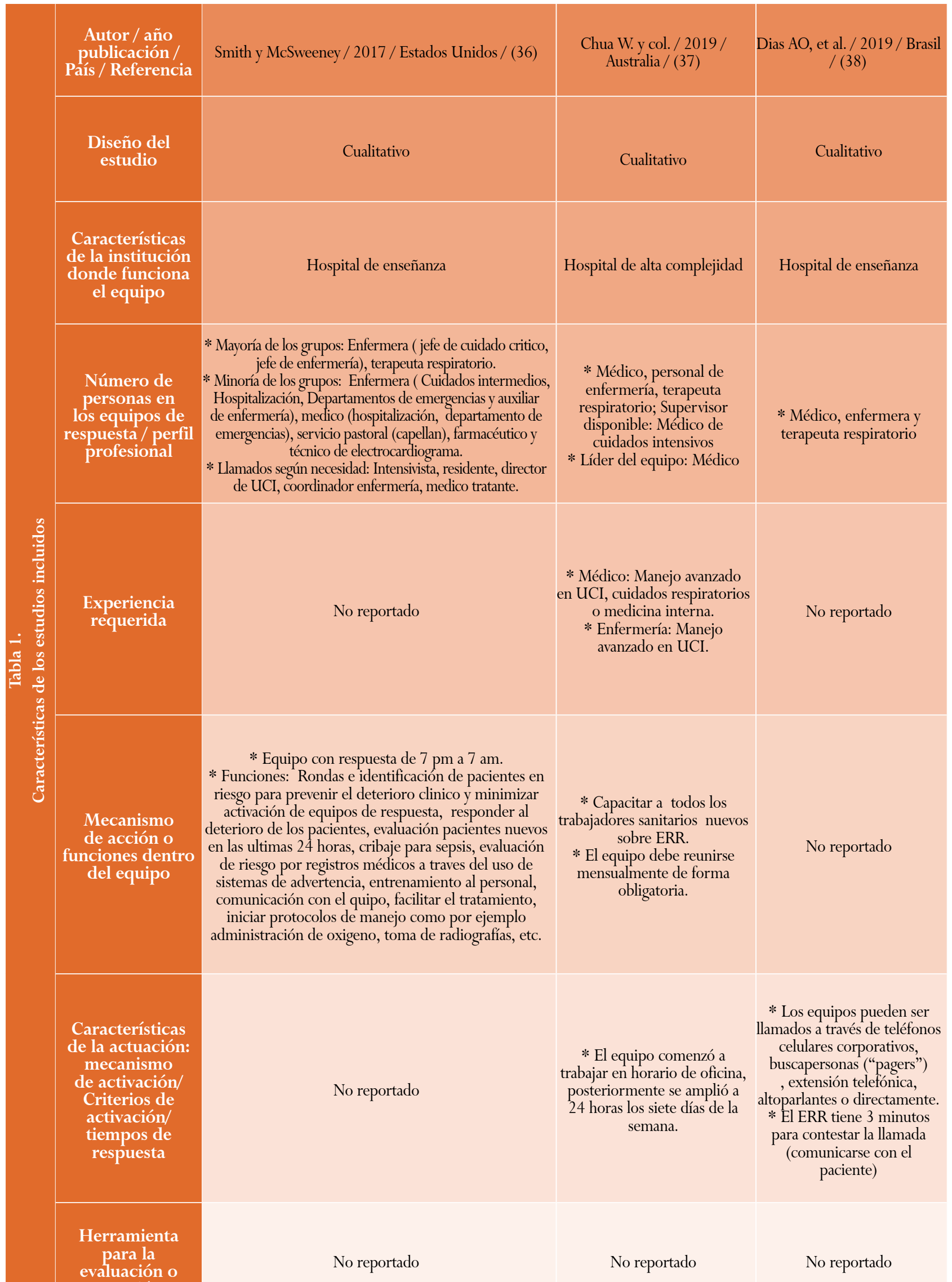




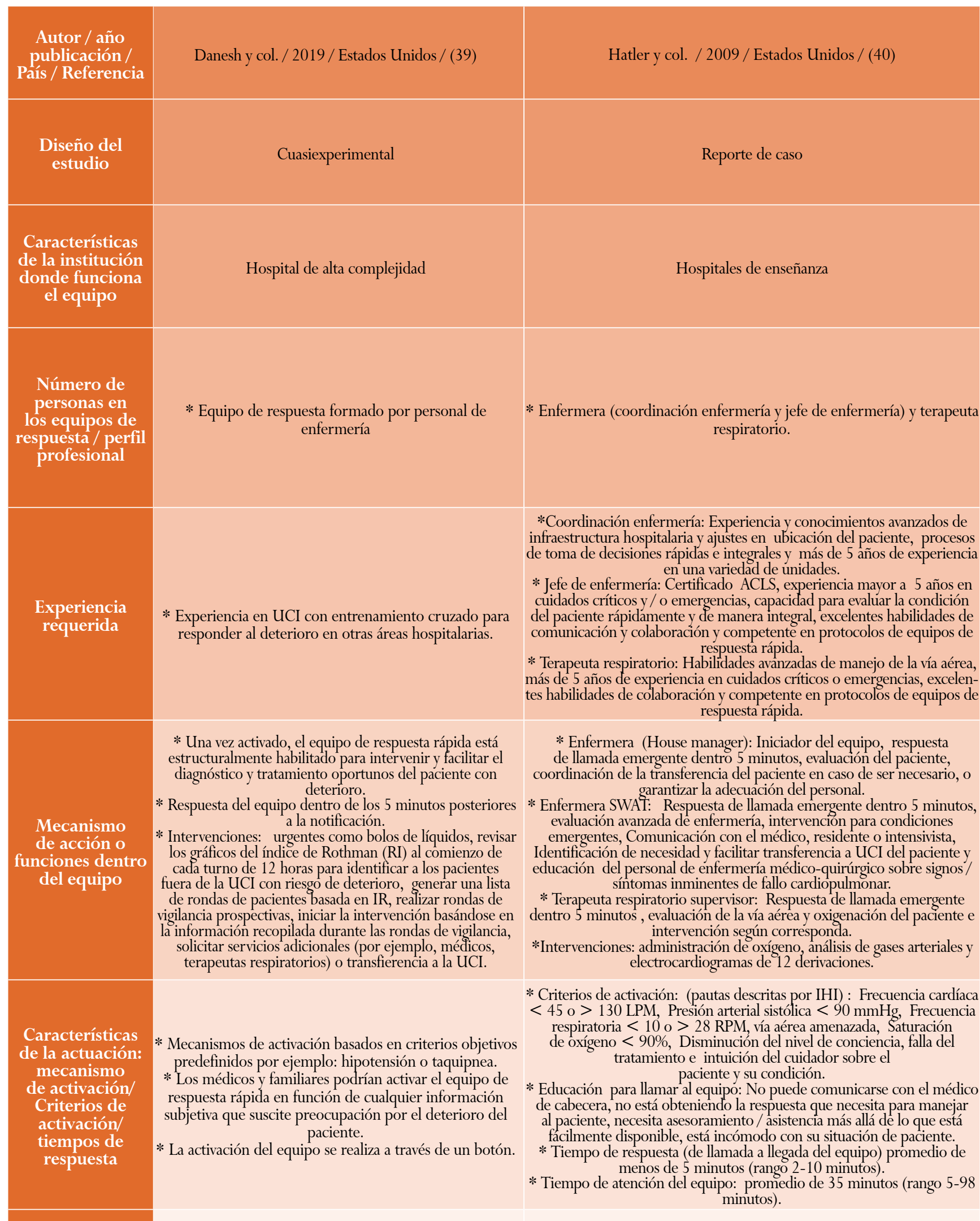

Las puntuaciones de alerta temprana (EWS; "Early Warning Scores") y el índice de Rothman se registran para evaluar los cambios de tendencia en la condición del paciente y sirven como desencadenantes de las intervenciones.
No reportado

\section{Herramienta \\ para la \\ evaluación o} atención

Continuación Tabla 1 


\begin{tabular}{|c|c|c|}
\hline $\begin{array}{c}\text { Autor / año } \\
\text { publicación / } \\
\text { País / Referencia }\end{array}$ & $\begin{array}{l}\text { Clifford Hughes y Col. / } 2014 \text { / Australia / } \\
\text { (41) }\end{array}$ & King y col. / 2006 / Estados unidos / (42) \\
\hline $\begin{array}{l}\text { Diseño del } \\
\text { estudio }\end{array}$ & Reporte de caso & Reporte de caso \\
\hline $\begin{array}{l}\text { Características } \\
\text { de la institución } \\
\text { donde funciona } \\
\text { el equipo }\end{array}$ & Hospital de alta complejidad & Hospital de alta complejidad \\
\hline $\begin{array}{l}\text { Número de } \\
\text { personas en } \\
\text { los equipos de } \\
\text { respuesta / perfil } \\
\text { profesional }\end{array}$ & No reportado & $\begin{array}{c}\text { * Día }(8 \text { am-5 pm): médico tratante (medico hospitalización capacitado } \\
\text { como internista, interno mayor, residente de medicina interna), } \\
\text { enfermera de cuidados críticos, jefe de enfemería, farmacéutico y } \\
\text { terapeuta respiratorio. } \\
\text { * Noche }(5 \mathrm{pm}-8 \mathrm{am}) \text { y fin de semana: Medico de hospitalización se } \\
\text { cambia por médico de cuidados críticos pulmonares, resto del equipo } \\
\text { igual. } \\
\text { * Líder del equipo: Médico. }\end{array}$ \\
\hline $\begin{array}{l}\text { Experiencia } \\
\text { requerida }\end{array}$ & $\begin{array}{l}\text { * Médicos de UCI con habilidades } \\
\text { avanzadas de soporte vital. }\end{array}$ & No reportado \\
\hline $\begin{array}{l}\text { Mecanismo } \\
\text { de acción o } \\
\text { funciones dentro } \\
\text { del equipo }\end{array}$ & $\begin{array}{l}\text { * El paciente en zona roja requiere la } \\
\text { intervención inmediata de los equipos de } \\
\text { respuesta. } \\
\text { * Los pacientes en las zonas amarillas deben } \\
\text { ser monitoreados por alguien del equipo de } \\
\text { admisión o local dentro de media hora. } \\
\text { * Intervenciones: Educación para mejorar } \\
\text { el juicio clínico sobre el reconocimiento de } \\
\text { deterioro. }\end{array}$ & $\begin{array}{c}\text { * Reuniones informativas semanales para revisar todos los eventos activados } \\
\text { de la semana anterior: identificar el problema que llevo a las activaciones, } \\
\text { seguimiento del paciente desde el evento clínico, evaluar el desempeño } \\
\text { del equipo y estrategias de "Quality impromevement" para mejorar la } \\
\text { efectividad del equipo. }\end{array}$ \\
\hline $\begin{array}{l}\text { Características } \\
\text { de la actuación: } \\
\text { mecanismo } \\
\text { de activación/ } \\
\text { Criterios de } \\
\text { activación/ } \\
\text { tiempos de } \\
\text { respuesta }\end{array}$ & $\begin{array}{l}\text { Los criterios para activar el equipo de respuesta } \\
\text { rápida son: frecuencia respiratoria } 6-10 \text { y } 25-29 \\
\text { LPM (zona amarilla, señales de alerta temprana), } \\
\leq 5 \text { y } \geq 30 \text { (zona roja, señales de alerta tardía); } \\
\text { Saturación de oxígeno (SpO2,\%) } 91-95 \text { (zona } \\
\text { amarilla), } \leq 90 \text { (zona roja): Frecuencia cardíaca } \\
41-50 \text { y } 120-139 \text { BPM (zona amarilla), } \leq 40 \text { y } \\
\geq 140 \text { BPM (zona roja); Presión arterial sistólica } \\
\text { (mm Hg) } 91-99 \text { y } 180-199 \text { (zona amarilla), } \leq 90 \\
\text { y } \geq 200 \text { (zona roja); Nivel de conciencia (escala } \\
\text { AVPU) verbal (zona amarilla), dolor o falta de } \\
\left.\text { respuesta (zona roja); y temperatura ( }{ }^{\circ} \mathrm{C}\right) \leq 35.5^{\circ} \\
\text { C y } \geq 38.5^{\circ} \mathrm{C} \text { (zona amarilla), criterio nulo (rojo). }\end{array}$ & $\begin{array}{l}\text { *Criterios de activación: } \\
\text { PULMONAR: Frecuencia respiratoria }<8 \text { o }>30 \text { RPM, disnea súbita de aparicón nueva y } \\
\text { prolongada }(>5 \text { minutos), saturación de oxigeno }<90 \% \text { ó nuevo requerimiento de }>50 \% \\
\text { oxigeno para preservar saturación }>85 \% \text {. } \\
\text { CARDIOVASCULAR: Dolor torácico que no responda a nitroglicerina o medico no disponi- } \\
\text { ble, frecuencia cardiaca }<40 \text { o }>140 \text { LPM sintomática, cualquier frecuencia cardiaca }>160 \\
\text { LPM, presión arterial sistólica }<80 \text { o }>200 \text { mmHg ó diastólica }>110 \mathrm{mmHg} \text { sintomática } \\
\text { (dolor torácico, cambio neurológico y disnea) ó cambio súbito del color de una extremidad. } \\
\text { NEUROLOGICO/PSIQUIATRICO: Perdida de conciencia súbita o colapso, administra- } \\
\text { ción de naloxona por sobredosis sin respuesta inmediata, inicio de letargia o dificultad para } \\
\text { despertar, convulsión, perdida súbita de movilidad en cara, brazos o piernas, agitación no } \\
\text { explicada }>10 \text { minutos ó intento de suicidio. OTROS: Trauma, accidente cerebrovascular, } \\
\text { perdida aguda y profusa de sangre, sangrado no controlado, sangrado en vía aérea, incapacidad } \\
\text { de contactar con el equipo tratante ó cualquier eventos adverso serio potencial. }\end{array}$ \\
\hline $\begin{array}{l}\text { Herramienta } \\
\text { para la } \\
\text { evaluación o } \\
\text { atención }\end{array}$ & $\begin{array}{l}\text { Sistema para identificar al paciente en } \\
\text { riesgo "Between the Flags" mediante la } \\
\text { monitorización de constantes vitales. }\end{array}$ & $\begin{array}{l}\text { * Cada evento es evaluado a través de un formulario estandarizado que incluye: } \\
\text { nombre del paciente, fecha, ubicación, miembros del equipo de atención, } \\
\text { diagnostico de ingreso y actual, razón para la activación : fue apropiada o no, } \\
\text { desenlace, causa del llamado, el equipo laboro sin problemas (en caso de } \\
\text { negativa especificar por que), se contacto al medico encargado del paciente. }\end{array}$ \\
\hline
\end{tabular}

Continuación Tabla 1 


\begin{tabular}{|c|c|c|c|}
\hline $\begin{array}{l}\text { Autor / año } \\
\text { publicación / } \\
\text { País / Referencia }\end{array}$ & $\begin{array}{l}\text { Danielis M., et } \\
\text { al./2021/Italia/ (43) }\end{array}$ & $\begin{array}{l}\text { Psirides y col. / } 2013 \text { / Nueva Zelanda/ } \\
\text { (44) }\end{array}$ & Wynn y col. / 2009 / Estados Unidos / (45) \\
\hline $\begin{array}{l}\text { Diseño del } \\
\text { estudio }\end{array}$ & Reporte de caso & Corte transversal & Corte transversal \\
\hline $\begin{array}{l}\text { Características } \\
\text { de la institución } \\
\text { donde funciona } \\
\text { el equipo }\end{array}$ & Hospital de enseñanza & Hospitales de alta complejidad & Hospital de enseñanza \\
\hline $\begin{array}{c}\text { Número de } \\
\text { personas en } \\
\text { los equipos de } \\
\text { respuesta / perfil } \\
\text { profesional }\end{array}$ & $\begin{array}{c}\text { * Anestesiólogo y } \\
\text { personal de enfermería } \\
\text { que laboran en la UCI, } \\
\text { ambos con dedicación } \\
\text { exclusiva. }\end{array}$ & No reportado & $\begin{array}{l}\text { * Médico, enfermera y terapeuta respiratorio, todos } \\
\text { con dedicación exclusiva. }\end{array}$ \\
\hline $\begin{array}{l}\text { Experiencia } \\
\text { requerida }\end{array}$ & $\begin{array}{c}\text { * Capacitación } \\
\text { proporcionada por } \\
\text { la institución en } \\
\text { prácticas técnicas } \\
\text { (por ejemplo, manejo } \\
\text { de vías respiratorias } \\
\text { y ventilación) y no } \\
\text { técnicas (por ejemplo, } \\
\text { habilidades de trabajo } \\
\text { en equipo) }\end{array}$ & No reportado & No reportado \\
\hline $\begin{array}{c}\text { Mecanismo } \\
\text { de acción o } \\
\text { funciones dentro } \\
\text { del equipo }\end{array}$ & $\begin{array}{l}\text { * El ERR está equipado } \\
\text { con un carro de } \\
\text { reanimación para } \\
\text { soporte vital avanzado } \\
\text { y otros procedimientos } \\
\text { urgentes (p. } \\
\text { Ej., Intubación } \\
\text { endotraqueal) } \\
\text { * El ERR llega a la } \\
\text { habitación, evalúa al } \\
\text { paciente, realiza el } \\
\text { tratamiento en el lugar } \\
\text { o analiza las opciones } \\
\text { de tratamiento con el } \\
\text { personal de turno. }\end{array}$ & No reportado & $\begin{array}{c}\text { * Primera fase de implementación: equipo de } 7 \text { PM } \\
\text { a } 7 \text { AM, poteriormente se extendió a } 24 \text { horas los } 7 \\
\text { días de la semana. } \\
\text { * Función del equipo: Respuesta temprana a signos } \\
\text { de deterioro del paciente como método para reducir } \\
\text { los eventos de código azul, ayudar al personal de } \\
\text { enfermería cuando la condición de un paciente se } \\
\text { deteriora. }\end{array}$ \\
\hline
\end{tabular}

* (1) Activación del equipo de respuesta rápida: Frecuencia respiratoria (bradipnea $<=8$ o $<=4$ RPM y taquipnea $>=$ $30,>=35$ ó $>=40 \mathrm{RPM}$ ), frecuencia cardiaca (taquicardia $>=120,>=130$

ó $>=140$ LPM y bradicardia $<=40$ ó

ERR es alertado a través de una línea telefónica exclusiva ubicada en la UCI; disponible las 24 horas del día, los 7 días de la semana.

activación

tiempos de

respuesta

\section{Herramienta} para la

evaluación o atención

Continuación Tabla 1 urinario (oliguria y poliuria), saturacion de oxigeno, flujo de oxígeno administrado, nivel de conciencia o vía aérea amenazada.
* (1) Se activa el llamado con la escala Early Warning Score $>=30>=7$ (sin embargo en todos los casos se realiza el llamado por un trastorno extremo en un solo parámetro.)

$<30$ LPM), presión sanguínea sistólica $<=$ personal de enfermería que tenga preocupación por $70 \mathrm{mmHg}$, temperatura (hipotermia $<=$ su paciente, este personal se describe como la primera $35^{\circ} \mathrm{C}$, hipertermia $\geq 38.1-38.6^{\circ} \mathrm{C}$ ), gasto línea de seguridad del paciente.

* La herramienta "Pre-RRT Patient Condition" es un instrumento de una página diseñado para recopilar datos pertinentes sobre la condición del paciente en las horas previas a la llamada del equipo de respuesta; diseñada para facilitar la identificación de los cambios en la condición del paciente en intervalos de 2 horas. 


\begin{tabular}{|c|c|c|}
\hline $\begin{array}{l}\text { Autor / año } \\
\text { publicación / } \\
\text { País / Referencia }\end{array}$ & $\begin{array}{c}\text { Ganju y col. / } 2019 \text { / Australia } \\
\text { / (46) }\end{array}$ & Mezzaroba y col. / 2016 / Brasil / (47) \\
\hline $\begin{array}{l}\text { Diseño del } \\
\text { estudio }\end{array}$ & Cohorte retrospectiva & Cohorte retrospectiva \\
\hline $\begin{array}{l}\text { Características } \\
\text { de la institución } \\
\text { donde funciona } \\
\text { el equipo }\end{array}$ & Hospital de enseñanza & Hospital de enseñanza \\
\hline $\begin{array}{c}\text { Número de } \\
\text { personas en } \\
\text { los equipos de } \\
\text { respuesta / perfil } \\
\text { profesional }\end{array}$ & No reportado & $\begin{array}{l}\text { * Medico de cuidados intensivos y terapeuta respiratorio. } \\
\text { * Por las noches la respuesta la ejerce el personal de servicios de emergencia. }\end{array}$ \\
\hline $\begin{array}{l}\text { Experiencia } \\
\text { requerida }\end{array}$ & No reportado & No reportado \\
\hline $\begin{array}{l}\text { Mecanismo } \\
\text { de acción o } \\
\text { funciones dentro } \\
\text { del equipo }\end{array}$ & No reportado & $\begin{array}{l}\text { * Equipo con respuesta de 7:00 am a 7:00 p.m., todos los días de la semana. } \\
\text { * Funciones: evaluaciones diarias (mañana y tarde) de pacientes críticos que no } \\
\text { fueron a UCI (pacientes con indicación de ingreso sin acceso inmediato por falta de } \\
\text { disponibilidad de camas): exámenes físicos diarios, revisar formulas médicas, verificar } \\
\text { resultados de pruebas y acompañar profesionales encargados del cuidado de estos } \\
\text { pacientes (evaluación hasta mejoría clínica o traslado). } \\
\text { *Intervenciones: Llamar al médico responsable de definir la terapia individual, solicitar } \\
\text { valoración fisioterapia, inserción de acceso venoso central, discutir limitaciones del } \\
\text { soporte terapéutico, indicar abordajes quirúrgicos, devolver a ventilación mecánica, } \\
\text { intubación endotraqueal o traqueostomía, aspiración a través de la cavidad oral, uso } \\
\text { de globo de Sengstaken-Blakemore, prescripción de: volumenes, drogas vasoactivas, } \\
\text { antibióticos y sedación, solicitud de pruebas: hematológicas y bioquímicas, radiografía de } \\
\text { tórax, cultivo de sangre, orina o de aspiración traqueal, electrocardiografía y tomografía } \\
\text { computarizada. }\end{array}$ \\
\hline $\begin{array}{l}\text { Características } \\
\text { de la actuación: } \\
\text { mecanismo } \\
\text { de activación/ } \\
\text { Criterios de } \\
\text { activación/ } \\
\text { tiempos de } \\
\text { respuesta }\end{array}$ & $\begin{array}{l}* \text { Criterios de activación: } \\
\text { Frecuencia cardiaca }>= \\
140 \text { o }<40 \text { LPM, presión } \\
\text { sanguínea sistólica }>=200 \\
\text { o }<90 \mathrm{mmHg} \text {, Frecuencia } \\
\text { respiratoria }>30 \text { o }<8 \text { RPM, } \\
\text { Saturación por pulsioximetría } \\
<90 \% \text {, nivel de conciencia } \\
\text { (Escala de sedación) solo } \\
\text { responde a estímulos físicos o } \\
\text { dolorosos }(>2)\end{array}$ & $\begin{array}{c}\text { * Criterios de activación: paro cardiorrespiratorio (código azul) y datos de inestabilidad } \\
\text { clínica (código amarillo); incluidos saturación de oxígeno }<90 \% \text {, frecuencia } \\
\text { respiratoria }<10 \text { o }>\text { a } 30 \text { RPM, presión arterial sistólica }<90 \text { ò }>180 \mathrm{mmHg} \text { con } \\
\text { síntomas, frecuencia cardíaca }<45 \text { ò }>125 \mathrm{LPM} \text {, disminución del nivel de conciencia, } \\
\text { convulsiones o preocupaciones serias del equipo médico con respecto al estado clínico } \\
\text { general del paciente. } \\
\text { * Tiempos de respuesta: dos minutos para código azul y cinco minutos para código } \\
\text { amarillo. }\end{array}$ \\
\hline
\end{tabular}

publicación

aís / Referencia

Diseño del

estudio

Características

de la institución

ende funciona
Número de

personas en

los equipos de

respuesta / perfil

profesional
Experiencia requerida
Mecanismo

de acción o

ciones dentro del equipo
Herramienta para la

evaluación o atención

Continuación Tabla 1

* Funciones: evaluaciones diarias (mañana y tarde) de pacientes críticos que no fueron a UCI (pacientes con indicación de ingreso sin acceso inmediato por falta de ( examenes físicos diarios, revisar formulas médicas, verificar pacientes (evaluación hasta mejoría clínica o traslado)

Intervenciones: Llamar al médico responsable de definir la terapia individual, solicitar valoración fisioterapia, inserción de acceso venoso central, discutir limitaciones de soporte terapéutico, indicar abordajes quirúrgicos, devolver a ventilación mecánica intubación endotraqueal o traqueostomía, aspiración a través de la cavidad oral, uso geno de Senostaken-Blakemore, prescripción de: volumenes, drogas vasoactivas, ntibióticos y sedación, solicitud de pruebas: hematológicas y bioquímicas, radiografía de tórax, cultivo de sangre, orina o de aspiración traqueal, electrocardiografía y tomografía computarizada. Frecuencia cardiaca $>=$ sanguínea sistólica $>=200$ $90 \mathrm{mmHg}$, Frecuencia (Escala de sedación) solo dolorosos $(>2)$

\section{Criterios de activación: paro cardiorrespiratorio (código azul) clínica (código amarillo); incluidos saturación de oxígeno $<90 \%$, frecuencia} 作

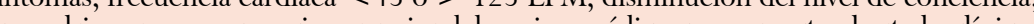
general del paciente. amarillo. 


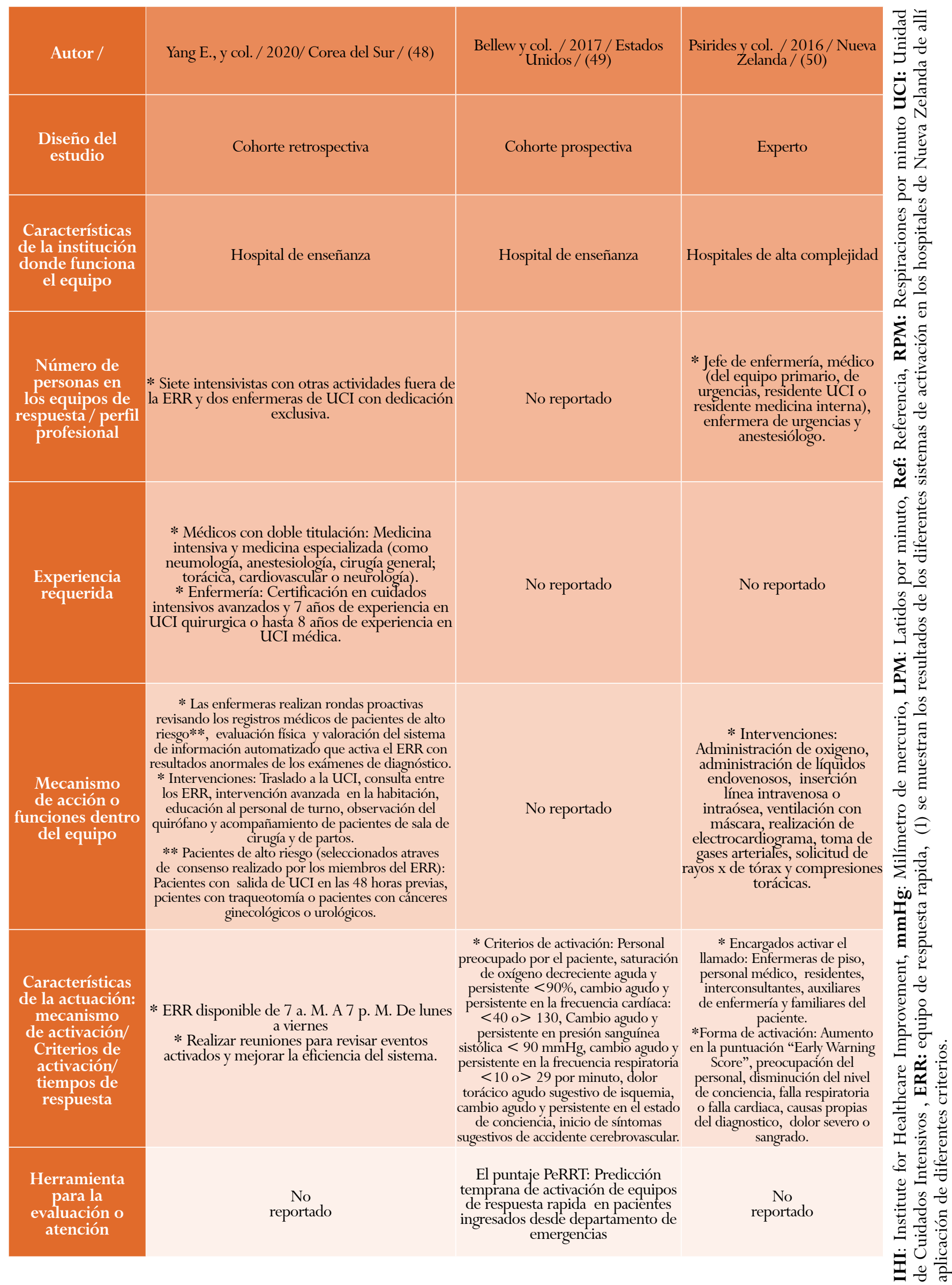


De los 15 estudios incluidos (40\%), seis fueron desarrollados en los Estados Unidos (36, 39, 40, 42, 45, 49), tres en Australia (20\%) (37, 41, 46), dos en Brasil $(13,3 \%)(38,47)$, dos en Nueva Zelanda $(13,3 \%)(44$, $50)$, uno en Corea del Sur (6,7\%) (48) y uno en Italia (43) (6,7\%). Los años de publicación fueron desde el 2006 hasta el 2021. De los 15 estudios, 9 se desarrollaron en hospitales universitarios o de enseñanza (60\%) $(36,38,40,43,45,46,47,48,49)$.

\section{Contexto de desarrollo de los estudios}

De los 15 estudios incluidos, 13 incorporaron ERR que funcionaran en servicios de hospitalización general (36-47, 50), un equipo trabajó específicamente en pacientes hospitalizados que ingresaron remitidos desde salas de reanimación (49) y uno observó pacientes en salas de cirugía y obstetricia, con otra categoría relevante: pacientes de alto riesgo (seleccionados mediante consenso por miembros del ERR); aquellos con estancia en UCI 48 horas antes, pacientes con traqueostomía o con cánceres ginecológicos o urológicos (48).

\section{Valoración de calidad metodológica}

Se asignó un puntaje de 6/8 estrellas a dos de cinco estudios de cohorte $(47,50)$ valorados con la herramienta NOS. Se consideró que el estudio de Psirides et al. (50) adolecía de sesgos, de datos incompletos y sesgo de selección dada la utilización de muestreo de conveniencia. Adicionalmente, el diseño del estudio no contempló la comparación entre centros de estudio. El estudio de Mezzaroba et al. (47), retrospectivo basado en registros electrónicos, tampoco realizó comparaciones. Por este motivo, no le fue asignado puntaje en las secciones de cohortes no expuestas y comparabilidad. Los estudios de Ganju (46), Yang (48) y Bellew (49) fueron considerados de alta calidad por el control de sesgos de selección, de factores de confusión en las comparaciones, y la valoración de desenlaces planteados.

Se encontró que todos los reportes de casos evaluados describen adecuadamente los ERR y obtuvieron una puntuación de 7/7 en el momento de la evaluación.
Todos los estudios cualitativos encontraron congruencia entre la perspectiva filosófica declarada, la pregunta u objetivos de investigación y la representación y análisis de datos con la metodología de investigación. Se incluyeron las voces de los participantes del RRT descritos. Sin embargo, no se hicieron declaraciones culturales, o teóricas ubicando a los investigadores o evaluando la relevancia de este tema en el estudio y viceversa.

Con el instrumento de lectura crítica y evaluación de estudios epidemiológicos transversales Berra S. et al. (31), el estudio de Psirides et al. (44) se obtuvo un alto puntaje en la validez interna y externa, y fue evaluado como de alta calidad. El estudio de Wynn et al. (45) se consideró como de validez interna intermedia dado el uso de herramientas no validadas para la medición de desenlaces principales, la pérdida de datos y la selección a conveniencia de la muestra para el análisis, lo cual en conjunto podría introducir sesgos de selección y compromete su validez externa. El estudio de Danesh et al. (39), evaluado con la herramienta de valoración crítica de JBI cumplió con 8 de los 10 criterios de calidad dispuestos. La naturaleza del estudio, de antes y después, impide declarar que haya habido un vínculo causal claro entre los efectos observados y la intervención, o que el efecto no se haya debido a otras variables diferentes de la intervención de interés, como lo declaran los autores en el apartado de limitaciones. Se consideró el análisis estadístico correcto, y que se tomaron medidas para evitar sesgos de información.

En el apéndice 2 se describe la información detallada sobre la evaluación de la calidad metodológica de los estudios incluidos.

\section{Estructura de ERR}

De 15 estudios incluidos, 11 describieron la estructura de los ERR (36-40, 42, 43, 45, 47, 48, 50). Los miembros del equipo incluyeron personal de enfermería, profesionales médicos, terapeutas respiratorios y otros. Ninguno de los estudios recomendó un número específico de miembros para el diseño de los ERR. 
De estos 11 estudios, 10 incluyeron personal de enfermería $(36,40,42,43,45,48,50)$. Un estudio describió un ERR compuesto únicamente por personal de enfermería (39). Cinco estudios describieron requerimientos de experiencia para personal de enfermería (37, 39, 40, 43, 48). Danesh et al. (39) describieron su experiencia en una UCI con capacitación cruzada de los miembros del personal para lidiar con el deterioro clínico que pudiera ocurrir fuera de las UCI. Hatler et al. describieron la inclusión de personal de enfermería en dos roles: coordinadoras de enfermería locales (House Manager) y como personal de enfermería con entrenamiento avanzado (SWAT Nurse). Como experiencia requerida para las coordinadoras locales, se describen conocimientos avanzados en infraestructura y procesos hospitalarios; experiencia de toma de decisiones rápida y completa; y 5 o más años de experiencia en diversas unidades y contextos hospitalarios. Como requisitos de experiencia para las enfermeras SWAT, describen la certificación de soporte vital cardiaco avanzado (ACLS), 5 o más años de experiencia en departamentos de cuidados intensivos o de emergencia; capacidades de evaluación clínica rápidas y completas; excelentes capacidades de comunicación y colaboración; y competencia en protocolos de ERR (40).

Nueve de los 11 estudios incluidos (36-38, 42, 43, $45,47,48,50)$ mencionaron la inclusión de médicos dentro de los ERR, describiendo los siguientes perfiles: médicos de urgencias o anestesiólogos $(36,43)$, intensivistas $(37,47)$, neumólogos y cardiólogos (36, 48), o médicos residentes de cuidados intensivos (42) y de medicina interna $(42,50)$. Dos estudios no especificaron el perfil de los médicos del equipo $(38,45)$. Tres estudios mencionaron requisitos de experiencia para ellos $(37,43,48)$, e incluyen manejo avanzado de UCI, cuidados respiratorios o medicina interna y habilidades no técnicas como el trabajo en equipo. Tres estudios dieron detalles sobre el rol de liderazgo dentro de los ERR. Chua et al. y King et al mencionan que el rol debe ser asumido por un médico $(37,42)$. Mezzaroba et al. mencionan que debe ser tomado por un intensivista (47).
Un estudio describe la inclusión de otros miembros en los ERR, estos son: farmacéuticos, técnicos en electrocardiograma y atención pastoral (36).

\section{Disponibilidad del equipo}

Siete de los 15 estudios incluyeron horas de trabajo de los TSR (36, 37, 42, 43, 45, 47, 48). Tres estudios informan ERR que funcionan las 24 horas del día, los siete días de la semana $(37,42,43)$. King et al. (42) describieron su composición según la hora del día, pasando de contratar a un médico internista de 08:00 am a 17:00 pm los días laborables, a un neumólogo especialista en cuidados intensivos de 17:00 pm a 08:00 am y los fines de semana.

El estudio de Wynn et al. (45) describió disponibilidad de las 19:00 pm a las 07:00 am durante la fase de implementación del ERR del estudio, que luego se extendió a las 24 horas del día, los 7 días de la semana. Mezzaroba et al. (47) describieron un ERR con horario de trabajo de 07:00 am a 19:00 pm que delegaba la respuesta de llamadas al personal de emergencia por la noche; sin embargo, este personal de emergencia no constituye en sí mismo un RRT (42). Dos estudios mencionaron la dedicación exclusiva de sus miembros al ERR $(43,45)$.

\section{Funcionamiento}

Diez de los 15 estudios incluidos mencionan funciones a realizar por el ERR (36, 37, 40, 42, 43, 45, 47, 48). Entre estos se mencionan los siguientes (sin ninguna jerarquía particular):

- Pacientes ingresados: rondas de identificación para evaluar el riesgo y prevenir el deterioro clínico, cribado de sepsis y evaluación del riesgo a través de sistemas de alerta digital automatizados en las historias clínicas electrónicas $(36,48)$.

- Respuesta temprana a los signos de deterioro clínico como una forma de reducir los eventos de código azul $(37,45)$.

- Evaluación dos veces al día de pacientes en condiciones críticas que no fueron ingresados en UCI (pacientes con indicación de traslado 
a UCI a los que no se les dio acceso inmediato por falta de cama) hasta que la condición del paciente mejore o sean trasladados a UCI (47).

- Capacitación del personal para la identificación oportuna y adecuada de los signos de deterioro clínico $(36,42,48)$.

- Apoyo del personal de enfermería cuando el estado de salud de un paciente se deteriora (40, 43, 45).

- Evaluación del desempeño del equipo a través de sesiones informativas que evalúan los eventos de activación ocurridos durante la semana pasada $(36,48)$.

Entre los temas tratados durante las sesiones informativas King et al. mencionan la identificación del detonante de activación, el seguimiento del paciente, la evaluación del desempeño del equipo y la evaluación de estrategias para mejorar la efectividad a través de una perspectiva de mejora continua de la calidad (42).

\section{Criterios de activación del ERR}

Doce de los 15 estudios mencionaron los criterios de activación del ERR (37, 39-42, 44-50). En general, la activación del equipo responde a un deterioro del estado de salud del paciente. Estos se manifiestan en la alteración de parámetros clínicos como frecuencia cardíaca (bradicardia o taquicardia), frecuencia respiratoria (taquipnea o bradipnea), disminución sostenida de la saturación de oxígeno en sangre, alteraciones de la presión arterial (hipo o hipertensión), alteraciones de la conciencia, sospecha de infarto de miocardio o evento cerebrovascular (37, $39,42,44,46,49)$ y variaciones de temperatura y diuresis (44). Estos parámetros se pueden observar en detalle en la Tabla 1, donde se muestran las variaciones en los rangos de referencia. Mezzaroba et al., por ejemplo, toman la bradipnea como criterio de activación cuando son menos de 10 respiraciones por minuto (rpm) (47), mientras que King et al., hacen lo mismo cuando es menos de $8 \mathrm{rpm}$ (42). De estos 12 estudios, solo King et al. consideraron la presentación de condiciones psiquiátricas como un criterio de activación para los ERR (42); entre estos se encuentran: letargo/dificultad para levantarse de la cama (de forma reciente), administración de naloxona después de sospecha de sobredosis sin respuesta inmediata, agitación inexplicable o intento de suicidio. Además, cuatro autores (37, 39, 40, 49, 50) describen la posibilidad de activar el ERR cuando no hay posibilidad de comunicación directa con el equipo tratante, si existe preocupación general por el estado del paciente, cuando se necesita asesoramiento adicional, o cuando el paciente presenta dolor severo o sangrado con características específicas.

\section{Personal responsable de la activación del ERR}

Cinco de los 15 estudios describen procedimientos específicos para la activación del ERR (37, 39 ,42, 48, 50). El personal de enfermería de hospitalización, los terapeutas respiratorios, los médicos, los servicios de interconsulta, los servicios auxiliares de atención, los cuidadores de pacientes y los familiares, se describen como capaces de activar el ERR. Ningún estudio indicó que el paciente inició la activación del ERR.

\section{Herramientas para facilitar el trabajo en los ERR}

Seis de los 15 estudios describieron el uso de herramientas en los ERR con diferentes fines: a) activar los ERR; y b) facilitar los procesos de cuidado e identificar cambios en la condición del paciente (39, 41, 42, 44, 49, 50).

Para activar los ERR, dos estudios reportaron que sus equipos estaban guiados por Sistemas de Puntaje de Alerta Temprana (Early Warning Score - EWS - Systems), con umbrales de $>=3$ y $>=7$ para iniciar el llamado $(44,50)$. Danesh et al. reportaron la aplicación del Índice de Rothman incorporado a Registros Médicos Electrónicos (Electronic Health Records), como componente del EWS (39). Este método incluye signos vitales, criterios paraclínicos, y valoraciones del sistema de enfermería para calcular un valor índice que representa la tendencia en la condición del paciente a través del tiempo, actuali- 
zándose hasta una vez cada hora. Otra herramienta reportada fue el sistema "Entre Banderas" (Between the Flags), el cual utiliza registros de signos vitales para determinar el requerimiento de intervención de un ERR (41).

Respecto al proceso de atención, King et al. mencionan el uso de un formato estandarizado que incluía: nombre del paciente, fecha, ubicación, miembros del equipo de ERR, diagnóstico de ingreso y de trabajo, causa de activación, relevancia de la causa, desenlace, dificultades durante la atención (si las hay), y si el equipo tratante fue contactado (42).

Un estudio desarrolló una herramienta llamada “Condición del Paciente Pre-ERR” (Pre-RRT Patient Condition) basada en los diagnósticos y signos vitales del paciente; esta se ubica junto a la cama del paciente, y fue diseñada para cuantificar el riesgo de activación de los ERR y ayudar en la toma de decisiones complejas relacionadas con la disposición de los pacientes. Además, el estudio describe la intención de facilitar la identificación de cambios en el estado de salud de los pacientes transferidos desde el departamento de urgencias, con un intervalo de aplicación de dos horas (49).

\section{Intervenciones de los ERR}

Para satisfacer las funciones previamente descritas, siete de los 15 estudios detallan intervenciones específicas llevadas a cabo por los ERR $(36,39,40$, 43, 47, 48, 49).

- Iniciación del protocolo de manejo y facilitación del tratamiento $(36,39,43,47)$.

- Comunicación con equipo tratante (36).

- Indicación de abordajes quirúrgicos (47, 48).

- Solicitud de consultas de especialistas $(39,47)$.

- Evaluación conjunta de limitaciones del soporte terapéutico $(47,48)$.

- Intervenciones relacionadas con soporte de oxigenación de los pacientes: administración de oxígeno $(36,40,49)$, ventilación por máscara (49), retorno a ventilación mecánica, intubación endotraqueal, traqueostomía, aspiración de vía aérea por cavidad oral y análisis de gases arteriales (40).
- Control hemostático en vías aéreas superiores por colocación de tubo Sengstaken-Blakemore (40).

- Inserción de acceso central o intraóseo (47, 49).

- Prescripción de medicamentos: agentes vasoactivos, antibióticos, agentes sedantes (47) y líquidos intravenosos $(39,47,49)$.

- Seguimiento clínico y paraclínico por solicitud de exámenes paraclínicos: exámenes bioquímicos y hematológicos; cultivos sanguíneos, de orina y de aspirados endotraqueales; electrocardiografía; tomografías axiales computarizadas; y estudios radiológicos (36, 40, 47, 49).

- Administración de compresiones torácicas $(43,49)$.

- Observación en el quirófano y salas de obstetricia (48).

\section{Tiempos de respuesta de los ERR}

Cuatro estudios reportaron tiempos de respuesta de los ERR (38, 40, 48). Se propone que el tiempo de respuesta al llamado sea de dos a cinco minutos (38-40, 48), y que el equipo se tome alrededor de 25 minutos para evaluar y definir conductas en los pacientes (40).

\section{DISCUSIÓN}

Esta revisión sistemática de alcance describió cómo se componen y funcionan los ERR, así como las herramientas que utilizan para facilitar su trabajo. Se identifica que la estructura de los ERR es multidisciplinaria (enfermeras experimentadas, médicos y terapeutas respiratorios principalmente), donde deben estar disponibles al menos 12 horas. En términos de funciones, la identificación temprana de pacientes con deterioro de la condición, especialmente en salas fuera de UCI, y pacientes con condiciones subyacentes o eventos desencadenantes que aumentan el riesgo de paro cardíaco e intervenciones rápidas multifacéticas que incluyen implementación de guías clínicas explícitas, tratamiento farmacológico, procedimientos cardiopulmonares, actividades de comunicación con el equipo tratante según el estado del paciente, 
seguimiento inicial, actividades de desarrollo de capacidades en la institución y continuas a la mejora de la calidad son parte de las funciones del RRT. Se dispone de herramientas para la activación, evaluación de los procesos asistenciales. Se encontraron dos estudios en América Latina. Por otra parte, solo uno menciona su utilización en los servicios de obstetricia. Las otras recomendaciones son aplicables a los servicios quirúrgicos en general, donde se incluyen las pacientes con cirugía ginecológica mayor o con factores de riesgo por edad mayor a 70 años, o comorbilidad previa, obesidad, entre otras $(51,52)$.

\section{Integridad y aplicabilidad}

Los autores realizaron una búsqueda exhaustiva en las principales bases de datos de la literatura gris e internacional. No se utilizaron límites con respecto a la fecha de publicación; sin embargo, colocamos límites de idioma (inglés, español y portugués) que podrían afectar la identificación de estudios realizados en otros países. Otra limitación es la no inclusión de revistas principales en pacientes de seguridad. Estas decisiones podrían resultar en algún sesgo de selección de los estudios, así como la selección de estudios por título y resumen por un solo investigador.

Con respecto a la aplicabilidad, no está claro que el enfoque de ERR funcione para EREND o eventos adversos, debido al hecho de que no se encontraron estudios en los que la población objetivo de ERR fueran específicamente pacientes con EREND; sin embargo, tampoco los excluye como población que se podría beneficiar de la disponibilidad de los ERR, ya que como lo menciona Berwick son muy útiles en situaciones de emergencia, como los errores de medicación, las infecciones asociadas al catéter o al respirador (21) que son considerados EREND, ya que surgen como consecuencia del cuidado provisto. De esta manera, muchos ERAND podrían terminar en un deterioro del paciente o un paro cardíaco y, en consecuencia, podrían detectarse y mitigar el daño mediante ERR. Por otra parte, solo se encontró un estudio que incluyó evaluación de ERR en servicios obstétricos, debido a que el enfoque de activación de código podría ser el más utilizado, especialmente en Latinoamérica (53). Vale la pena recordar que la activación del código responde a una situación específica donde cada persona conoce el rol que juega, y que se agrupa cuando ocurre el código (21), pero el ERR se constituye como un equipo estable.

\section{Calidad de los estudios incluidos}

La evaluación de la calidad de las cohortes mostró una calidad aceptable en los estudios de cohortes, transversales y de informes de casos. Sin embargo, el conjunto de pruebas se considera de certeza baja porque proviene de estudios observacionales. La inclusión de estudios cualitativos que den cuenta de las voces de los actores relevantes favorece la identificación de barreras y ventajas de la implementación del ERR. Una barrera probable en la implementación es la limitación de recursos y personal que dificultaron la adopción de medidas como la contratación de un equipo de ERR independiente y de dedicación exclusiva.

\section{CONCLUSIÓN}

La estructura y las funciones del ERR están claramente descritas, lo que permite que sean ensamblados en hospitales de alta complejidad. Se deben realizar más investigaciones sobre los beneficios y riesgos del uso de los ERR para mitigar los daños en pacientes con EREND y comparar la efectividad y seguridad entre la activación de códigos y las estrategias de ERR en los servicios de obstetricia.

\section{FINANCIACIÓN}

Esta investigación fue financiada con recursos del Ministerio de Ciencia y Tecnología para el proyecto de jóvenes investigadores, código 202010014648 de 2018.

\section{Papel de los patrocinadores del estudio}

Minciencias es una agencia del gobierno colombiano que apoya la investigación fundamental y aplicada en Colombia. Minciencias otorgó autonomía al Grupo de Evaluación de Políticas y Tecnologías en Salud para el desarrollo del presente estudio y no participó en su diseño o desarrollo . 


\section{INTERCAMBIO DE DATOS Y DISPONIBILIDAD DE DATOS DE APOYO}

Todos los datos utilizados para los análisis en este estudio pueden y serán compartidos a solicitud. Aprobación ética y consentimiento para participar Esta revisión del alcance se llevó a cabo siguiendo un protocolo desarrollado previamente que está disponible a solicitud.

\section{REFERENCIAS}

1. Emelia B, Salim S, Clifton W, Alanna M, Alexander R, Susan C, et al. Heart Disease and Stroke Statistics-2018 Update: a report from the American Heart Association. American Heart Association. 2018; 137(12): p. e67-e492. https://doi.org/10.1161/ CIR.0000000000000558

2. Wallmuller C, Meron G, Kurkciyan I, Schober A, Stratil P, Sterz F. Causes of in-hospital cardiac arrest and influence on outcome. Resuscitation. 2012; 83(10):1206-1211. https://doi.org/10.1016/j.resuscitation.2012.05.001

3. Sinha SS, Sukul D, Lazarus JJ, Polavarapu V, Chan PS, Neumar RW, et al. Identifying important gaps in randomized controlled trials of adult cardiac arrest treatments: a systematic review of the published literature. Circ Cardiovasc Qual Outcomes. 2016; 9(6):749-756. https://doi.org/10.1161/CIRCOUTCOMES.116.002916

4. Holmberg MJ, Ross CE, Fitzmaurice GM, Chan PS, Duval-Arnould J, Grossestreuer AV, et al. Annual Incidence of Adult and Pediatric In-Hospital Cardiac Arrest in the United States. Circ Cardiovasc Qual Outcomes. 2019; 12(7): e005580. https://pubmed. ncbi.nlm.nih.gov/31545574/

5. Estrada-Orozco K, Gaitán-Duarte H, Moreno S, MorenoChaparro J. Reportable hospital events: incidence and contributing factors in the surgery service of a high complexity hospital in Bogotá, Colombia, 2017. Rev. colomb. anestesiol. 2019; 47(1): p. 5-13. https://doi. org/10.1097/CJ9.0000000000000091

6. James JT. A new, evidence-based estimate of patient harms associated with hospital care. J
Patient Saf. 2013; 9(3):122-8. https://doi.org/10.1097/ PTS.0b013e3182948a69

7. Brennan TA, Leape LL, Laird NM, Hebert L, Localio AR, Lawthers AG, et al. Incidence of Adverse Events and Negligence in Hospitalized Patients. N Engl J Med. 1991; 324(6):370-6. https://doi.org/10.1056/ NEJM199102073240604

8. Wilson RM, Runciman WB, Gibberd RW, Harrison BT, Newby L, Hamilton JD. The Quality in Australian Health Care Study. Med J Aust. 1995; 163:458-7. https://doi. org/10.5694/j.1326-5377.1995.tb124691.x

9. Schwendimann R, Blatter C, Dhaini S, Simon M, Ausserhofer D. The occurrence, types, consequences and preventability of in-hospital adverse events - a scoping review. BMC Health Serv Res. 2018; 18(1):521. https:// doi.org/10.1186/s12913-018-3335-z

10. Gaitán DH, Eslava SJ, Garzón OCD, Rubio JA, Forero GJ, Valbuena LG, et al. Tamizaje de eventos adversos en atención obstétrica y del puerperio en el Instituto Materno Infantil de Bogotá, Colombia. 2002 -2003. Rev. Colomb. Obstet. Ginecol. 2005; 56(1):18-7. https:// doi.org/10.18597/rcog.555

11. Dzakpasu S, Deb-Rinker P, Arbour L, Darling EK, Kramer MS, Liu S, et al. Severe maternal morbidity surveillance: Monitoring pregnant women at high risk for prolonged hospitalization and death. Paediatr Perinat Epidemiol. 2020; 34(4):427-439. https://doi.org/10.1111/ ppe. 12574

12. Instituto Nacional De Salud Colombia. Boletín Epidemiológico Nacional. Morbilidad Maternal Extrema. Febrero 21 de 202. Disponible en: https://www.ins.gov. co/buscador-eventos/BoletinEpidemiologico/2021_Boletin_epidemiologico_semana_8.pdf

13. Vélez-Maya MA, Grillo-Ardila CF, Higuera-R IL, Molano D. Caracterización de la mortalidad materna temprana en Bogotá. Estudio de vigilancia epidemiológica de casos centinela. Ginecol Obstet Mex. 2019; $87(7): 425-435$.

14. Villalba-Toquica C del P, Martínez-Silva PA. Morbilidad materna extrema en la Clínica Universitaria Colombia, Bogotá (Colombia), 2012-2013. Rev. Colomb. Obstet. Ginecol. 2014; 65(4):290-6. https://doi. org/10.18597/rcog.32 
15. Mahshid N, Ahmad S, Nahid M, Afshin F. Sudden cardiac arrest during cesarean section - a possible case of amniotic fluid embolism. Middle East J Anaesthesiol. 2009; 20(2):315-7

16. Alki M, Liu S, Leon JA, Baghirzada L. Epidemiology of cardiac arrest during hospitalization for delivery in Canadá: a nationwide study. Anesth Analg 2017; 124(3):890-7. https://doi.org/10.1213/ANE.0000000000001877

17. Gaitán-Duarte H, Eslava-Schmalbach J, RodríguezMalagón N, Forero-Supelano V, Santofimio-Sierra D, Altahona H. Incidence and preventability of adverse events in patients hospitalised in three Colombian hospitals during 2006. Rev Salud Pública. 2008;10(2):215-26. https://doi.org/10.1590/S012400642008000200002

18. Devita MA, Bellomo R, Hillman K, Kellum J, Rotondi A, Teres D. Findings of the first consensus conference on medical emergency teams. Crit Care Med. 2006; 34(9):2463-78. https://doi.org/10.1097/01. CCM.0000235743.38172.6E

19. Gaitán D. H. Los eventos adversos en la atención en salud. Rev. Colomb. Obstet. Ginecol. 2008; 59(4):270 -272. https://doi.org/10.18597/rcog.389

20. Andersen LW, Holmberg MJ, Berg KM, Donnino MW, Granfeldt A. In-Hospital Cardiac Arrest: A Review. JAMA. 2019; 321(12):1200-1210. https://doi.org/10.1001/ jama.2019.1696

21. Berwick DM, Calkins DR, McCannon CJ, Hackbarth AD. The 100,000 lives campaign: setting a goal and a deadline for improving health care quality. JAMA. 2006; 295(3):324-7. https://doi.org/10.1001/jama.295.3.324

22. MERIT study investigators. Introduction of the medical emergency team (MET) system: a cluster-randomised controlled trial. Lancet. 2005; 365: 2091-2097. https:// doi.org/10.1016/S0140-6736(05)66733-5

23. Chan PS, Jain R, Nallmothu BK, Berg RA, Sasson C. Rapid Response Teams: A Systematic Review and Meta-analysis. Arch Intern Med. 2010;170(1):18-26. https://doi.org/10.1001/ archinternmed.2009.424

24. Winters BD, Weaver SJ, Pfoh ER, Yang T, Pham JC, Dy SM. Rapid-response systems as a patient safety strategy: a systematic review. Ann Intern Med. 2013; 158: 417-25. https;/doi. org/10.7326/0003-4819-158-5-201303051-00009
25. Maharaj R, Raffaele I, Wendon J. Rapid response systems: a systematic review and meta-analysis. Crit Care. 2015;19(1):254. https://doi.org/10.1186/s13054-0150973-y

26. Solomon RS, Corwin GS, Barclay DC, Quddusi SF, Dannenberg MD. Effectiveness of rapid response teams on rates of in-hospital cardiopulmonary arrest and mortality: A systematic review and meta-analysis. J Hosp Med. 2016; 11(6):438-45. https://doi.org/10.1002/jhm.2554

27. Peters MDJ, Godfrey C, McInerney P, Munn Z, Tricco AC, Khalil, H. Chapter 11: Scoping Reviews (2020 version). In: Aromataris E, Munn Z (Editors). JBI Manual for Evidence Synthesis, JBI, 2020. https://doi.org/10.46658/ JBIMES-20-12

28. Wells GA, Shea B, O'Conell D, Peterson J, Welch V, Losos M, et al. The Newcastle-Ottawa Scale (NOS) for assessing the quality of nonrandomised studies in meta-analyses. 2000. Disponible en: http://www.ohri.ca/programs/clinical_epidemiology/oxford.asp

29. Lockwood C, Munn Z, Porrit K. Qualitative research synthesis: methodological guidance for systematic reviewers utilizing meta-aggregation. Int J Evid Based Heal. 2015; 13(3):179-87. https://doi.org/10.1097/ XEB.0000000000000062

30. Moola S, Munn Z, Tufanaru C, Aromataris E, Sears K, Sfetcu R, et al. Chapter 7: Systematic reviews of etiology and risk. In: Aromataris E, Munn Z (Editors). Joanna Briggs Institute Reviewer's Manual. The Joanna Briggs Institute, 2017.

31. Berra S, Elorza-Ricart JM, Estrada MD, Sánchez E. Instrumento para la lectura crítica y la evaluación de estudios epidemiológicos transversales. Gaceta Sanitaria. 2008; 22(5): 492-7. https://doi. org/10.1157/13126932

32. Tufanaru C, Munn Z, Aromataris E, Campbell J, Hopp L. Chapter 3: Systematic reviews of effectiveness. In: Aromataris E, Munn Z (Editors). Joanna Briggs Institute Reviewer's Manual. The Joanna Briggs Institute, 2017.

33. Higgins JP, Altman DG, Gøtzsche PC, Jüni P, Moher D, Oxman AD, et al. The Cochrane Collaboration's tool for assessing risk of bias in randomised trials. BMJ. 2011; 343: d5928. https://doi.org/10.1136/bmj.d5928 
34. Sandelowski M, Barroso J, Voils CI. Using qualitative metasummary to synthesize qualitative and quantitative descriptive findings. Res Nurs Health. 2007;30(1):99111. https://doi.org/10.1002/nur.20176

35. Tricco AC, Lillie E, Zarin W, O’Brien KK, Colquhoun H, Levac D, et al. PRISMA Extension for Scoping Reviews (PRISMA-ScR): Checklist and Explanation. Ann Intern Med. 2018; 169(7):467. https://doi. org/10.7326/M18-0850

36. Smith PL, McSweeney J. Organizational Perspectives on Rapid Response Team Structure, Function, and Cost: A Qualitative Study. Dimensions of Critical Care Nursing. 2017;36:3-13. https://doi.org/10.1097/ DCC.0000000000000222

37. Chua WL, Legido-Quigley H, Jones D, Hassan NB, Tee A, Liaw SY. A call for better doctor-nurse collaboration: A qualitative study of the experiences of junior doctors and nurses in escalating care for deteriorating ward patients. Aust Crit Care. 2020; 33(1):54-61. https:// doi.org/10.1016/j.aucc.2019.01.006

38. Dias AO, Bernardes A, Chaves LDP, Sonobe HM, Grion CMC, Haddad MCFL. Critical incidents as perceived by rapid response teams in emergency services. Rev Esc Enferm USP. 2020; 54: e03595. https://doi.org/10.1590/ s1980-220x2018027903595

39. Danesh V, Neff D, Jones TL, Aroian K, Unruh L, Andrews $\mathrm{D}$, et al. Can proactive rapid response team rounding improve surveillance and reduce unplanned escalations in care? A controlled before and after study. Int J Nurs Stud. 2019; 91:128-33. https://doi.org/10.1016/j. ijnurstu.2019.01.004

40. Hatler CW, Mast D, Bedker D, Johnson R, Corderella J, Torres J, et al. Implementing a rapid response team to decrease emergencies outside the ICU: one hospital's experience. Medsurg Nurs. 2009;18(2):84-90.

41. Hughes C, Pain C, Braithwaite J, Hillman K. "Between the flags": Implementing a rapid response system at scale. BMJ Quality and Safety. BMJ Publishing Group; 2014;23:7147. https://doi.org/10.1136/bmjqs-2014-002845

42. King E, Horvath R, Shulkin DJ. Establishing a rapid response team (RRT) in an academic hospital: one year's experience. J Hosp Med. 2006;1(5):296-305. https://doi.org/10.1002/jhm.114
43. Danielis M, Destrebecq A, Terzoni S, Palese A. Critical Incidents Involving the Medical Emergency Team: A 5-Year Retrospective Assessment for Healthcare Improvement. Dimens Crit Care Nurs. 2021;40(3):186-191. https:// doi.org/10.1097/DCC.0000000000000473

44. Psirides A, Hill J, Hurford S. A review of rapid response team activation parameters in New Zealand hospitals. Resuscitation. 2013;84(8):1040-4. https:// doi.org/10.1016/j.resuscitation.2013.01.022

45. Wynn JD, Engelke MK, Swanson M. The front line of patient safety: Staff nurses and rapid response team calls. Qual Manag Health Care. 2009;18(1):40-7. https:// doi.org/10.1097/01.QMH.0000344592.63757.51

46. Ganju A, Kapitola K, Chalwin R. Modifications to predefined rapid response team calling criteria: prevalence, characteristics and associated outcomes. Crit Care Resusc. 2019;21(1):32-38.

47. Mezzaroba AL, Tanita MT, Festti J, De Maio Carrilho CMD, Cardoso LTQ, Grion CMC. Evaluation of the five-year operation period of a rapid response team led by an intensive care physician at a university hospital. Rev Bras Ter Intensiva. 2016;28(3):278-84. https:// doi.org/10.5935/0103-507X.20160045

48. Yang E, Lee H, Lee SM, Kim S, Ryu HG, Lee HJ, et al. Effectiveness of a daytime rapid response system in hospitalized surgical ward patients. Acute Crit Care. 2020;35(2):77-86. https://doi.org/10.4266/ acc. 2019.00661

49. Bellew SD, Cabrera D, Lohse CM, Bellolio MF. Predicting Early Rapid Response Team Activation in Patients Admitted From the Emergency Department: The PeRRT Score. Acad Emerg Med. 2017;24(2):216-25. https://doi.org/10.1111/acem.13077

50. Psirides AJ, Hill J, Jones D. Rapid response team activation in New Zealand hospitals - A multicentre prospective observational study. Anaesth Intensive Care. 2016;44(3):391-7. https://doi. org/10.1177/0310057X1604400314

51. Ben-Ami I, Vaknin Z, Schneider D, Halperin R. Perioperative morbidity and mortality of gynecological oncologic surgery in elderly women. Int J Gynecol Cancer. 2006;16(1):452-7. https://doi.org/10.1111/ j.1525-1438.2006.00478.x 
52. Behbehani S, Suarez-Salvador E, Buras M, Magtibay P, Magrina J. Mortality Rates in Laparoscopic and Robotic Gynecologic Oncology Surgery: A Systemic Review and Meta-analysis. J Minim Invasive Gynecol. 2019;26(7):1253-1267.e4. https://doi.org/10.1016/j. jmig.2019.06.017

53. Vélez-Álvarez GA, Agudelo-Jaramillo B, Gómez-Dávila JG, Zuleta-Tobón JJ. Código rojo: guía para el manejo de la hemorragia obstétrica. Rev. Colomb. Obstet. Ginecol. 2009;60(1):34-8. https://doi.org/10.18597/ rcog. 352

\section{CONTRIBUCIÓN DE LOS AUTORES}

Juliana Rincón-López: participó en el desarrollo del protocolo, búsqueda, selección de los estudios, extracción de los datos y elaboración del documento final.

Diego Andrés Larrotta-Castillo: participó en la selección de los estudios, extracción de los datos y elaboración del documento final.

Kelly Estrada-Orozco: participó en el desarrollo del protocolo, elaboración y revisión del documento final.

Hernando Guillermo Gaitán-Duarte: participó en el desarrollo del protocolo, elaboración y revisión del documento final.

\section{Conflicto de intereses: ninguno de los autores declara conflicto de intereses. Todos los autores de-} claran su independencia de los financiadores del estudio. 